\title{
Nuevos desafíos para repensar la formación del profesorado ecuatoriano
}

\author{
New challenges to rethink \\ the training of ecuadorian teachers
}

Recibido: 18 de noviembre de 2012

Aceptado: 2 de diciembre de 2012

De la misma manera en que no puedo ser profesor sin sentirme capacitado para enseñar correctamente y bien los contenidos de mi disciplina tampoco puedo, por otro lado, reducir mi práctica docente a la mera enseñanza de esos contenidos.

Ese es tan solo un momento de mi actividad pedagógica. Tan importante como la enseñanza de los contenidos es mi testimonio ético al enseñarlos.

Es la decencia con que lo hago.

Es la preparación científica revelada sin arrogancia, al contrario con humildad.

Es el respeto nunca negado al educando, a su saber "hecho de experiencia" que busco superar junto a él.

(Paulo Freire, 1996)

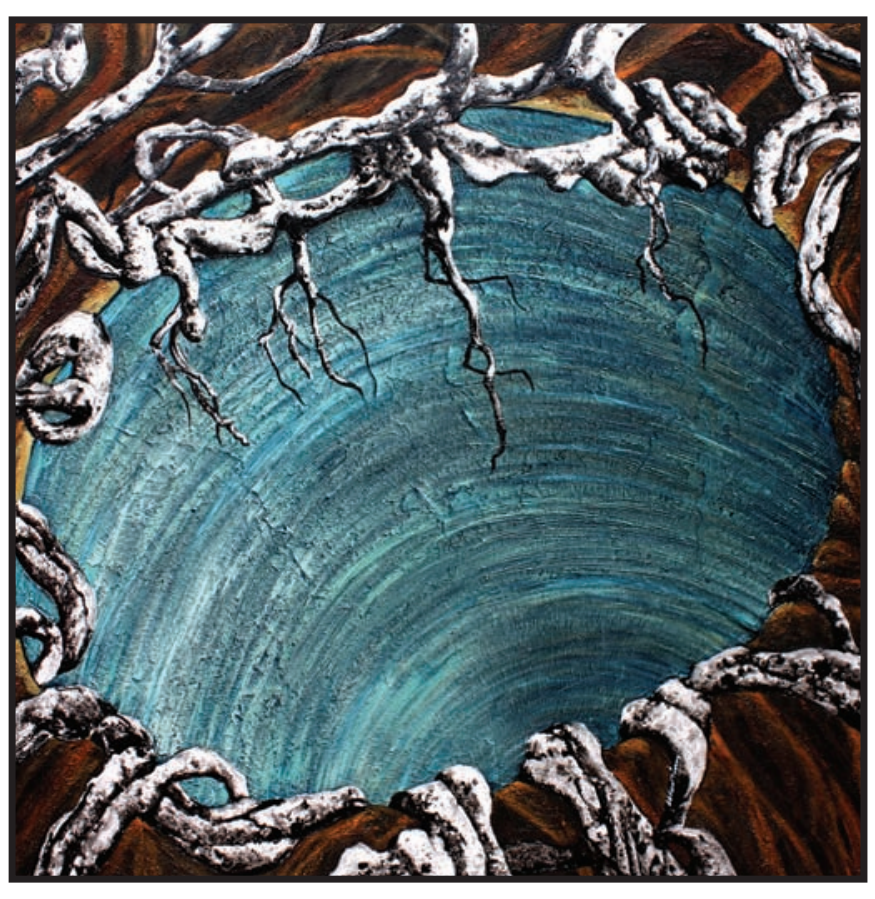

A cielo abierto. $146 \times 146 \mathrm{~cm}$ 


\section{Resumen}

La formación del docente ecuatoriano ha estado tradicionalmente ligada a los lineamientos de la política pública y del currículo nacional que han definido el quehacer docente, el trabajo de aula y lo que las instituciones de formación inicial docente deben priorizar en lo que respecta a la formación del profesorado. Los últimos años presentan grandes transformaciones en el sector educativo, además de la revalorización del trabajo docente. Se plantean nuevos desafíos formativos, ya no únicamente desde la perspectiva del docente como ejecutor de un currículo, sino como actor fundamental del proceso educativo. Es necesario repensar la formación de los profesores desde y para la reflexión crítica y la investigación, para la reflexión sobre el currículo, sobre sus propias prácticas y la pertinencia de éstas en relación a los contextos donde se desarrollan.

\section{Palabras clave}

Formación de profesores, política educativa, docencia.

\section{Política pública y formación docente en la década de los 1990}

La formación del docente de educación básica en el Ecuador se encuentra bastante ligada a la política educativa pública del país y en cierta forma a los requerimientos del ejercicio docente provenientes de las exigencias de las reformas educativas. Según Fernanda Saforcada, la década de los noventa significó en varios países de la región, en el campo del conocimiento y de la educación:

procesos de privatización y de mercantilización [...] como resultantes de las políticas educativas neoliberales desarrolladas en el contexto del Consenso de Washington y de las reformas del Estado impulsadas desde los organismos internacionales y los propios gobiernos de turno $[. .$.$] la privatización manifiesta en la$ delegación de la responsabilidad pública en la esfera privada que gestiona la escuela desde sus intereses particulares y la mercantilización que transfiere tres elementos del mercado a la edu-

\begin{abstract}
Training Ecuadorian teachers has traditionally been linked to public policy and national curricula that have defined the task of teaching, classroom work, and what basic teacher training institutions should prioritize on when it comes to training teachers. Recent years have seen major changes in the educational sector, as well as a revaluation of the teaching trade. New training challenges arise, not only from the teacher's perspective as executor of the curricula, but as a key player in the educational process. We need to rethink the training of teachers to and from a critical reflection and research point-of-view, in order to reflect on the curricula, their own experience, and their relevance in relation to the framework in which they develop.
\end{abstract}

\section{Keywords}

Teacher training, educational policy, teaching.

cación: libre elección de las familias sobre el tipo de educación que prefieren para sus hijos, autonomía de las escuelas para desarrollar sus propios proyectos educativos y competencia para desarrollar mejores proyectos educativos. [...] Estos dos procesos, privatización y mercantilización, se articulan con la tendencia a la primacía del derecho de propiedad por sobre los derechos sociales, en este caso, el derecho a la educación. (2009)

Las consecuencias de esta política, coloca a los usuarios del sistema en una grave desigualdad de condiciones reflejada en la inequidad de recursos y en diferencias en términos de calidad de las ofertas educativas; esto se aprecia tanto en infraestructura física escolar como en los resultados de los logros de los aprendizajes. Asimismo, es importante señalar que la década de los noventa para el caso de América Latina, y por tanto del Ecuador, es significativa si se analiza la política educativa y las reformas de educación que de éstas se desprenden: 
Se fundamentan en una vigorosa descentralización de funciones y responsabilidades en el ámbito educacional [...] se propone repasar el fondo público hacia niveles cada vez más micro, evitando así la interferencia "perniciosa" del centralismo estatal; se desarticulan los mecanismos unificados de negociación con las organizaciones gremiales de los trabajadores de la educación [...] se flexibilizan las formas de contratación y las retribuciones salariales de los colectivos docentes. Pero, por otro lado, y al mismo tiempo, se lleva a cabo una no menos poderosa estrategia centralizadora fundada en: a) el desarrollo de programas nacionales de evaluación de los sistemas educativos altamente centralizados en su diseño e implementación (básicamente, pruebas de rendimiento aplicadas a la población estudiantil); b) el diseño hipercentralizado de reformas curriculares a partir de las cuales establecer los contenidos básicos de un Currículum Nacional; y, c) la implementación de programas nacionales de formación de profesores permitan la actualización de los docentes según el plan curricular establecido en la citada reforma. (Gentili, 2009)

En el Ecuador las reformas educativas de este período, se concretizan en nuevos instrumentos curriculares de alcance nacional que resultan determinantes en el trabajo docente y en su formación. Las reformas del currículo configuran los procesos formativos, las instituciones formadoras, universidades e institutos pedagógicos, y direccionan sus proyectos académicos de formación docente en función de lo que el docente debe trabajar en el aula. De esta manera, la concepción del currículo como orientador de la práctica educativa es la premisa, si bien no la única, pero sí una muy importante para definir las actividades docentes, los lineamientos del proceso de enseñanza aprendizaje, además de lo que se enseña y se aprende y el cómo se enseña y se aprende. Desde esta perspectiva, el currículo de educación básica y sus concreciones definen aquello que el futuro docente requerirá conocer y hacer para su trabajo, y desde allí se han direc- cionado las propuestas formativas iniciales y los programas de formación en trabajo.

La "Reforma Curricular Consensuada de Educación Básica” del Ecuador del año 1996', expresa las prescripciones para la educación básica nacional. La reforma vino acompañada por capacitaciones y por textos escolares. La empresa editorial privada se ocupó de brindar capacitación docente en relación al nuevo currículo y al uso de sus textos en los procesos educativos. Se evidencia en estas prácticas la delegación al sector privado, la capacitación docente.

Paralelamente, en esta misma década y como consecuencia de las conquistas alcanzadas por el movimiento indígena, luego de la creación de la DINEIB (Dirección Nacional de Educación Intercultural Bilingüe) en 1988, se gesta un currículo específico que aporta a la consolidación de la educación propia para los indígenas. De esta manera, el país contaría con dos modelos educativos para la educación básica, por un lado el sistema educativo oficial o denominado "hispano ${ }^{2 \text { ", }}$ y por otro lado, el MOSEIB 3 que fue sin duda un importantísimo aporte para la educación de los pueblos indígenas.

De esta forma, tanto las prescripciones del currículo nacional en el caso del sistema educativo hispano y del currículo de educación intercultural bilingüe, orientan los proyectos y procesos de formación docente. Actualmente, el Ecuador cuenta con instituciones formadoras de docentes que deben atender a los requerimientos del sistema de educación "hispano" e intercul-

1 La reforma curricular extendió el período de obligatoriedad de la educación básica de 6 a 10 años. Se autodefinió como consensuada por un proceso participativo de consulta y socialización con empresarios, académicos, docentes.

2 Se utiliza la denominación de sistema hispano, aquel que se desarrolla en contextos mestizos de educación formal, con dependencia administrativa de las instancias nacionales y generales de la dirección nacional de educación. El sistema intercultural bilingüe, por su parte depende administrativa y operativamente de la dirección nacional de educación intercultural bilingüe. De esta manera rigen en el país dos sistemas paralelos de educación.

3 El MOSEIB es el Modelo del Sistema de Educación Intercultural Bilingüe. 
tural bilingüe. Para el año 2007 funcionan en el Ecuador "cinco institutos pedagógicos de formación docente intercultural bilingüe (sierra: Cañar y Chimborazo; Amazonía: Sucumbíos, Pastaza y Morona Santiago) y dieciséis direcciones provinciales de EIB en el país (Esmeraldas, Imbabura, Pichincha, Cotopaxi, Tungurahua, Bolívar, Chimborazo, Cañar, Azuay, Loja, Sucumbíos, Orellana, Napo, Pastaza, Morona Santiago y Zamora Chinchipe)" (Velez, 2008), ubicadas geográficamente en aquellas provincias con mayor población indígena.

El caso particular de la Universidad Politécnica Salesiana como institución formadora inicial docente no escapa a esta doble necesidad del sistema educativo ecuatoriano. Actualmente, la UPS cuenta con dos carreras encargadas de la formación docente: la Carrera de Pedagogía y la Carrera de Educación Intercultural Bilingüe:

La Carrera de Pedagogía se crea en 1998 y atiende a una población mayoritariamente mestiza y que habita en el sector urbano y, la Carrera de Educación Intercultural Bilingüe por su parte, surge por la necesidad de contar con una instancia que forme a los docentes que laborarán en las Instituciones de Educación Intercultural Bilingüe $[. .$.$] atiende$ a un importante número de estudiantes en su mayoría indígenas y campesinos de las provincias de Cotopaxi, Chimborazo, Pichincha, Bolívar, Imbabura y Morona Santiago. Las cinco primeras con un alto número de población kichwa y la última con población mayoritariamente achuar. (Villagomez, 2012)

Es importante destacar el aporte y compromiso de las dos carreras en la formación del profesorado ecuatoriano, en especial en la formación de los docentes de los pueblos indígenas donde la presencia de la UPS ha sido fundamental:

El programa les ha ofrecido las herramientas necesarias para implementar un enfoque intercultural en su trabajo del día a día en el aula. Cuando decimos enfoque intercultural nos referimos a aquella perspectiva pedagógica que apunta a que los estudiantes, por un lado, conozcan y valoren los elementos del ámbito de su cultura propia, y que, por otro, conozcan la cultura que no les es propia y se apropien de aquellos elementos que consideran necesarios. (Iza \& Granda, 2012)

\section{La revalorización del sector educativo desde las políticas públicas}

Los últimos años evidencian transformaciones en las políticas educativas no solo del Ecuador sino también de la región:

La nueva política no refiere a un simple cambio electoral sino a un denominador común político en la región caracterizado por la oposición al Consenso de Washington y la adscripción a ideas y programas político tendientes a recomponer algunos de los más agudos efectos sociales, políticos e institucionales generados por aquella política. (Feldfeber, 2010)

En el Ecuador, a partir de los últimos cinco años se evidencia un proceso interesante en la política educativa pública tendiente a la revalorización del sector educativo y la recuperación de la rectoría del estado sobre la educación y el sistema educativo, a partir de lineamientos constitucionales y legales y desde la comprensión de la educación como un derecho en el marco del Buen Vivir:4

4 “El Buen Vivir es un principio constitucional basado en el Sumak Kawsay [...] El Buen Vivir y la Educación interactúan de dos modos. Por una parte el derecho a la educación es un componente esencial del Buen Vivir, en la medida en que permite el desarrollo de las potencialidades humanas y como tal garantiza la igualdad de oportunidades para todas las personas. Por otra parte, El Buen Vivir es eje esencial de la educación, en la medida en que el proceso educativo debe contemplar la preparación de los futuros ciudadanos para una sociedad inspirada en los principios del Buen Vivir, es decir 
Una señal significativa es la revalorización del sector educativo, después de un diagnóstico crítico a su calidad y equidad. Pocas veces la educación aparece tan nítidamente valorada como clave del desarrollo y la vocación social. La apuesta es total, y supera al sector para integrar un discurso de Gobierno. La opción por la educación se acompaña de nueva normativa, Plan Decenal, respaldo y estabilidad de autoridades, y dotación de recursos. (Luna \& Astorga, 2011)

El nuevo proyecto del Estado ecuatoriano, la aprobación de la Constitución del año 2008, la puesta en vigencia de las nuevas políticas educativas públicas del Ecuador, los lineamientos del Plan Nacional de Desarrollo, el nuevo marco legal del sistema educativo nacional desde el Registro Oficial de la Ley Orgánica de Educación Intercultural (LOEI) publicada en el año 2011 y el nuevo reglamento de la LOEI recientemente publicado en el mes de agosto de 2012, plantean importantes implicaciones en las prácticas educativas nacionales y por tanto, nuevos desafíos para el trabajo y la formación docente. Como Fabara afirma: "La Ley reconoce y da una alta valoración al trabajo del profesorado, como un factor esencial para lograr la calidad de la educación" (2011).

La LOEI determina una nueva arquitectura del sistema educativo del país, organizado, según se expresa en el Artículo 343, en un solo "sistema nacional de educación [...] tendrá como centro el sujeto que aprende, y funcionará de manera flexible y dinámica, incluyente, eficaz y eficiente. El sistema nacional de educación integrará una visión intercultural acorde a la diversidad geográfica, cultural y lingüística del país, y el respeto a los derechos de las comunidades, pueblos y nacionalidades" (Constitución de la República del Ecuador, 2008).

Lo mencionado implica una nueva organización del sistema educativo ecuatoriano que

una sociedad democrática, equitativa, inclusiva, pacífica, promotora de la interculturalidad, tolerante con la diversidad, y respetuosa de la naturaleza" (Ministerio de Educación, 2013). reconoce y le da el carácter de interculturalidad a la educación y al sistema educativo en general. Además señala la vigencia de un solo currículo nacional y en general una visión renovada de la educación. En el reglamento de Ley, se explicita la obligatoriedad de los currículos nacionales y su aplicación. Las adaptaciones curriculares comprendidas como complementariedades se realizan según los requerimientos culturales y peculiaridades de las instituciones. Con respecto a la formación docente, este mismo reglamento prevé procesos de formación permanente.

Asimismo, el Plan Decenal de Educación, otorga importancia a la formación docente, y entre sus objetivos estratégicos referentes al talento humano señala: "Renovar la formación inicial del personal docente, capacitar al personal administrativo y contribuir a mejorar su calidad de vida" (Ministerio de Educación, 2006). Este mismo documento plantea como Política 7: "La revalorización de la profesión docente, desarrollo profesional, condiciones de trabajo y calidad de Vida." Esta política se justifica en este documento con la siguiente afirmación:

Un factor que contribuye significativamente en los procesos de mejoramiento de la calidad de la educación es el docente, por ello la importancia de contribuir a su desarrollo profesional, mejorar las condiciones de trabajo y su calidad de vida. En la actualidad la formación inicial docente es débil y desactualizada; no existe un sistema integral y sostenido de desarrollo profesional, lo que ha provocado desvalorización del rol docente y poco reconocimiento social y económico a la profesión docente. (Ministerio de Educación, 2006)

De esta forma, el mismo Estado ecuatoriano reconoce las debilidades de la formación inicial de los docentes, así como también la necesidad de su formación para el desarrollo profesional y en trabajo.

En el año 2011, en la publicación del informe de acompañamiento al Plan Decenal de Educación se reconoce explícitamente que: 
El nivel de formación docente presenta algunos desafíos de mejora. De acuerdo con el AMIE al año 2010, del total de docentes de educación regular, aproximadamente el 7,3\% a escala nacional tiene título de Bachillerato en Ciencias de la Educación, el 65,9\% presenta título de nivel superior docente (3er nivel), el $6,2 \%$ título de posgrado (4to nivel), y el 20,6\% de los docentes no ha actualizado su nivel de formación docente. En cuanto a la mejora de la formación docente, desde el año 2009, el Ministerio de Educación puso en marcha el Sistema Integral de Desarrollo Profesional Educativo (Síprofe). Este sistema pretende incidir en la formación inicial docente, mejorar la oferta de formación continua, y proveer acompañamiento a los docentes y directivos. Sin embargo, ha existido mayor énfasis en la formación continua, a través de la organización de diversos cursos de orientación pedagógica, que presentan una duración de entre 10 y 60 horas. [...] pero se percibe que la capacidad de este sistema para abastecer la demanda de cursos de actualización y formación docente es limitada, y no suficiente. Por otra parte, es necesario que se realice un seguimiento de los resultados de estos programas. (Grupo Faro, 2011)

De la misma manera, y de acuerdo a la política sexta del Plan Decenal de Educación, el Ministerio de Educación del Ecuador implementa el Sistema Nacional de Evaluación y Rendición Social de Cuentas con el objetivo de realizar "el monitoreo de la calidad de la educación que brinda el sistema educativo ecuatoriano y la definición de políticas que permitan mejorar los procesos de enseñanza y aprendizaje" (Ministerio de Educación del Ecuador, 2012). Uno de los componentes a evaluar es precisamente el desempeño docente "que permitirá promover acciones didáctico-pedagógicas que favorezcan los procesos de aprendizaje de los estudiantes, y el mejoramiento de la formación inicial docente, así como su desarrollo profesional" (Ibid, 2012). Estos procesos de evaluación del desempeño docente han sido inéditos en nuestro país, han sido motivo de debate y discusión, han recibido el apoyo de unos sectores y el rechazo de otros, pero lo más complejo es quizás reconocer a través de ellos, la deficiente formación del profesorado:

Los resultados de las evaluaciones aplicadas a 4.885 docentes de la costa del país en 2009, demuestran que menos de la cuarta parte de los docentes evaluados tienen un desempeño satisfactorio, lo que significa que hay necesidad de implementar procesos de gran aliento en la formación continua y asumir una política prioritaria en la formación inicial de los docentes. (Fabara, 2011)

\section{Nuevos desafíos para la formación del profesorado}

Los desafíos para la formación docente se ubican ya no únicamente desde la perspectiva del docente como ejecutor de un currículo sino como actor fundamental del proceso educativo. Es necesario repensar la formación docente desde y para reflexión crítica y la investigación; para la reflexión sobre el currículo y sobre sus propias prácticas y la pertinencia de éstas en relación a los contextos donde se desarrollan: “...y nos preguntamos en qué medida el desplazamiento de las demandas de profesionalización a un modelo que propone el desarrollo profesional, supone nuevas formas de concebir e implementar las políticas en materia de formación, en la que los docentes dejan de ser el 'objeto' de la política para transformase en sujetos centrales en su construcción" (Angus, 1999).

Es indiscutible la relación entre formación inicial y en carrera y el desempeño profesional, así como también es indiscutible la relación entre desempeño docente y calidad de los aprendizajes. Por lo tanto, elevar la calidad de la formación docente es indispensable y a la par también es indispensable la revalorización del rol social del docente y la generación de políticas de desarrollo profesional.

Repensar los talentos humanos que se requieren para la educación exige repensar cuál 
es ese nuevo docente que el Ecuador necesita. El proceso formativo requiere recuperar la visión de los contextos escolares donde sucede el hecho educativo. Es importante además repensar la formación docente también desde lo plurinacional y la diversidad cultural.

Se requiere una "formación inicial donde logren los futuros profesores apropiarse del capital cultural de su grupo y certificar su saber profesional, que deberá caracterizarse por sólidos conocimientos disciplinarios, didácticos y pedagógicos" (Matus, 2012). Pero además de ello, dotar al docente de las herramientas necesarias para cuestionar sus prácticas y para innovarlas, dotarlo de las capacidades para aprender continuamente, para aprender de los otros y con los otros. "El momento fundamental en la formación permanente de los profesores es el de la reflexión crítica sobre la práctica. Es pensando críticamente la práctica de hoy o la de ayer como se puede mejorar la próxima" (Freire, 1996).

Desde esta reflexiones se pretende aportar al debate educativo en lo que refiere a formación docente, por lo que cobran actualidad las interrogantes: ¿qué se debe considerar como fundamental en la formación del docente?, ¿cuáles son los perfiles docentes que requiere el trabajo y la práctica docente?, ¿cuáles son los desafíos para las instituciones formadoras?, entre otras.

\section{Bibliografía}

Constitución de la República del Ecuador. 2008. Ecuador.

Angus, W. P.

1999. De la profesionalización al desarrollo profesional: algunas notas para pensar la política de formación docente. En D. Andrade Oliveira, \& M. Feldfeber, Nuevas regulaciones educativas en América Latina (p. 86). Lima: Fondo Editorial Universidad de Ciencias y Humanidades.

Fabara, E.

2011. Formación docente en el Ecuador. Estado del Arte. Quito, Ecuador: IESALC.
Farfán, $M$.

2008. Experiencias del Programa Académico Cotopaxi, Formación en Educación Intercultural Bilingüe de la Universidad Politécnica Salesiana (Ecuador). En D. Mato (Coord.), Diversidad Cultural $e$ Interculturalidad en Educación Superior. Caracas, Venezuela: IESALC.

Feldfeber, $\mathrm{M}$.

2010. De la profesionalización al desarrollo profesional: algunas notas para pensar las políticas de formación docente. En A. D. Dalida, \& M. Feldfeber, Nuevas regulaciones educativas en América Latina. Políticas $y$ procesos del trabajo docente (p. 84). Lima: Fondo Editorial Universidad de Ciencias y Humanidades.

Freire, P.

1996. Pedagogía de la autonomía. Saberes necesarios para la práctica educativa. México D. F.: Siglo Veintuno Editores.

Gentili, P., et al.

2009. Política de privatización, espacio público y educación en América Latina. Buenos Aires: Consejo Latinoamericano de Ciencias Sociales (CLACSO).

Grupo Faro.

2011. Educiudadanía. Recuperado el 6 de septiembre, 2012, de: http://www.grupofaro.org

Iza, A., \& Granda, S.

2012. Los Salesianos, la educación superior y los pueblos indígenas. El caso del Programa Académico Cotopaxi. En L. Vásquez, et al., La Presencia Salesiana en el Ecuador. Quito, Ecuador: Abya-Yala.

Luna, M., \& Astorga, A.

2011. Educación 1950-2012. Reformas inconclusas, nudos recurrentes, nuevos desafíos. En B. Adriá, \& M. Luna, Estado del País. Informe Cero. Ecuador 1950-2010. Quito: Activa.

Martínez, D.

2001. Abriendo el presente de una modernidad inconclusa: Treinta años de estudio de trabajo docente. Presentado durante el XXIII Congreso Internacional de LASA, 6-8 de septiembre, 2001, Washington D. C.

Matus, L.

2012. La construcción de una identidad docente. ¿Un desafío para la política educa- 
tiva? Santiago de Chile: RED ESTRADO.

Ministerio de Educación del Ecuador.

2013. Educación para la Democracia y el Buen Vivir. Recuperado el 7 de enero, 2013, de: http://www.conocimiento.gob.ec/educacion-para-la-democracia-y-el-buen-vivir/

Ministerio de Educación del Ecuador.

2012. Sistema Nacional de Evaluación. Recuperado el 16 de septiembre, 2012, de: http://www.educacion.gob.ec/profesionales/ sistema-nacional-evaluacion-p.html

Ministerio de Educación.

2006. Plan Decenal de Educación 2006-2015. Recuperado el 7 de septiembre, 2012, de: http://www.educación.gob.ec

Montaluisa, L.

2011. Plan Analítico de Educación e Interculturalidad. Quito.

Saforcada, F.

2009. Alambrando el bien común: Conocimiento, educación y derechos sociales en los procesos de privatización y mercantilización de las últimas décadas. En P. Gentili, et al. (Coords.), Politicas de privatización, espacio público y educación en América Latia (p. 371). Buenos Aires: Homo Sapiens.

Universidad Politécnia Salesiana

2004. Licenciatura en Ciencias de la Educación, Mención Pedagogía. Propuesta Unificada. Cuenca.

Vélez, C.

2008. Trayectoria dela Educación Intercultural en el Ecuador. Revista Educación y Pedagogía, 10, 103-112. Medellín: Universidad de Antioquia, Facultad de Educación.

Villagomez, M. S.

2012. La interculturalidad en el formación docente, el caso de la UPS como institución formadora. Presentado en IX Seminario Internacional Red Estrado, 18-20 de julio, 2012, Chile.

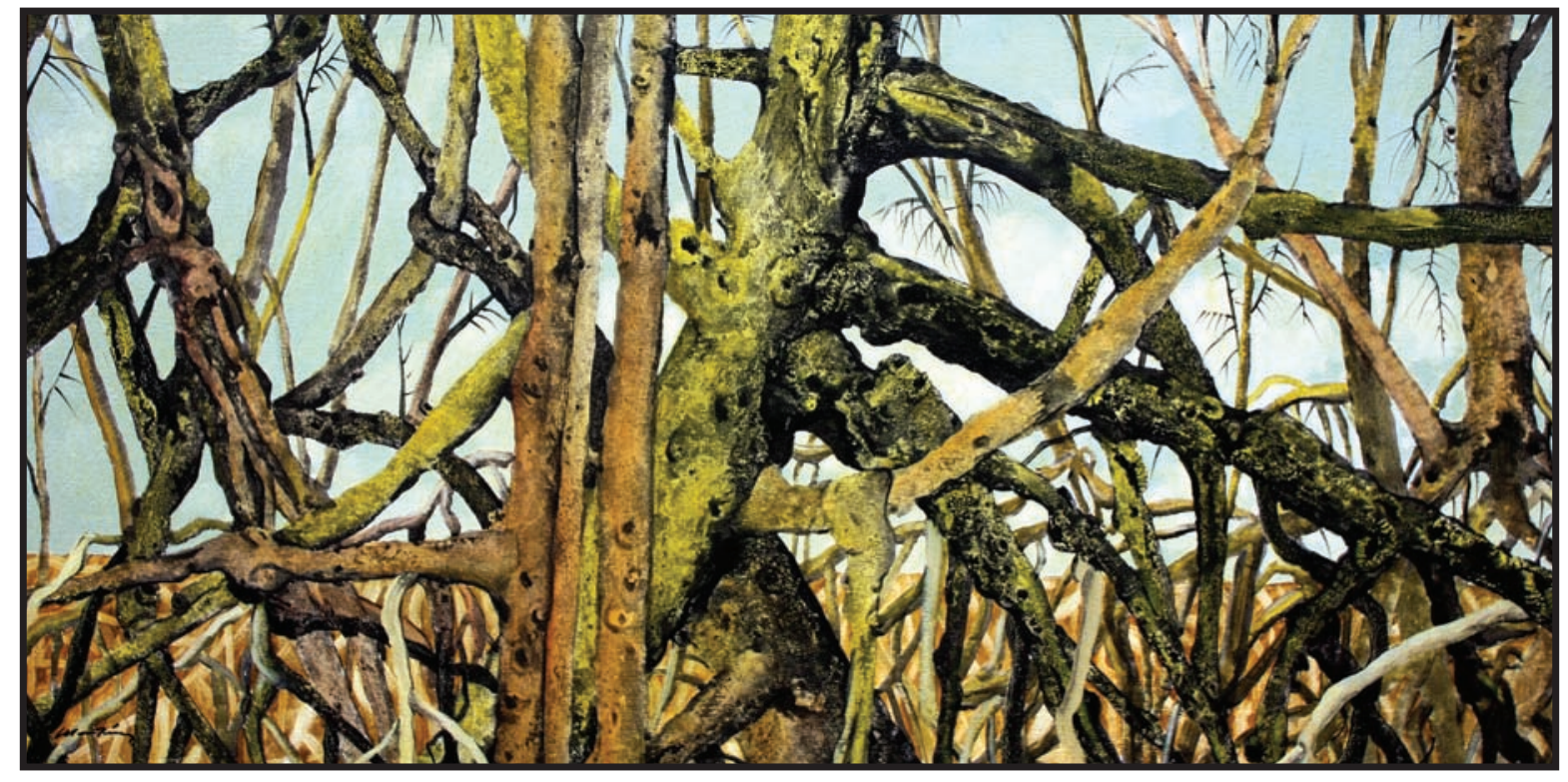

Luces en el manglar. $46 \times 92 \mathrm{~cm}$ 